\title{
Activation of sonic hedgehog signaling enhances cell migration and invasion by induction of matrix metalloproteinase-2 and -9 via the phosphoinositide-3 kinase/AKT signaling pathway in glioblastoma
}

\author{
LIANG CHANG ${ }^{1}$, DAN ZHAO ${ }^{2-4}$, HUI-BIN LIU $^{2-4}$, QIU-SHI WANG $^{2-4}$, PING ZHANG $^{1}$, CHEN-LONG LI $^{1}$, \\ WEN-ZHONG DU ${ }^{1}$, HONG-JUN WANG ${ }^{1}$, XING LIU $^{1}$, ZHI-REN ZHANG $^{2-4}$ and CHUAN-LU JIANG ${ }^{1}$ \\ Departments of ${ }^{1}$ Neurosurgery, ${ }^{2}$ Clinical Pharmacy and ${ }^{3}$ Cardiology, The Second Affiliated Hospital, \\ Harbin Medical University; ${ }^{4}$ Key Laboratories of the Education Ministry for Myocardial \\ Ischemia Mechanism and Treatment, Harbin, Heilongjiang 150086, P.R. China
}

Received October 30, 2014; Accepted July 28, 2015

DOI: $10.3892 / \mathrm{mmr} .2015 .4229$

\begin{abstract}
Aberrant hedgehog signaling contributes to the development of various malignancies, including glioblastoma (GBM). However, the potential mechanism of hedgehog signaling in GBM migration and invasion has remained to be elucidated. The present study showed that enhanced hedgehog signaling by recombinant human sonic hedgehog N-terminal peptide (rhSHH) promoted the adhesion, invasion and migration of GBM cells, accompanied by increases in mRNA and protein levels of matrix metalloproteinase-2 (MMP-2) and MMP-9. However, inhibition of hedgehog signaling with cyclopamine suppressed the adhesion, invasion and migration of GBM cells, accompanied by decreases in mRNA and protein levels of MMP-2 and -9. Furthermore, it was found that MMP-2- and MMP-9-neutralizing antibodies or GAM6001 reversed the inductive effects of rhSHH on cell migration and invasion. In addition, enhanced hedgehog signaling by rhSHH increased AKT phosphorylation, whereas blockade of hedgehog signaling decreased AKT phosphorylations. Further experiments showed that LY294002, an inhibitor of phosphoinositide-3 kinase (PI3K), decreased rhSHH-induced
\end{abstract}

Correspondence to: Professor Zhi-Ren Zhang, Department of Clinical Pharmacy, The Second Affiliated Hospital, Harbin Medical University, 246 Xuefu Road, Harbin, Heilongjiang 150086, P.R. China

E-mail: zhirenzh@yahoo.com

Professor Chuan-Lu Jiang, Department of Neurosurgery, The Second Affiliated Hospital, Harbin Medical University, 246 Xuefu Road, Harbin, Heilongjiang 150086, P.R. China

E-mail: jcl_6688@163.com

Key words: hedgehog pathway, phosphoinositide-3 kinase, AKT, matrix metalloproteinase-2, matrix metalloproteinase-9, glioblastoma, migration, invasion upregulation of MMP-2 and -9. Finally, the protein expression of glioblastoma-associated oncogene 1 was positively correlated with levels of phosphorylated AKT as well as protein expressions of MMP-2 and -9 in GBM tissue samples. In conclusion, the present study indicated that the hedgehog pathway regulates GBM-cell migration and invasion by increasing MMP-2 and MMP-9 production via the PI3K/AKT pathway.

\section{Introduction}

Glioma, the most common type of primary neoplasm of the brain, is the main cause of brain tumor-associated mortality. It can be divided into four sub-types according to the World Health Organization's classification of central nervous system tumors published in 2007 (1). Glioblastoma (GBM), the most malignant type of glioma, accounts for more than half of all gliomas in adults (2). Despite enormous efforts in the development and application of GBM therapies, the prognosis of patients with GBM remains poor due to the highly migratory and invasive nature of GBM cells $(3,4)$. The identification of the molecular mechanisms underlying the aggressive biological behavior of GBM may aid in the development of novel effective therapeutics with the aim of improving the prognosis of patients with GBM.

The hedgehog signaling pathway is essential for embryonic patterning and cancer development (5-7). The hedgehog protein family includes desert hedgehog, Indian hedgehog and sonic hedgehog $(\mathrm{SHH})$. Among them, $\mathrm{SHH}$ is the most widely expressed and the most potent protein. Once SHH binds to its receptor Patched 1, the transmembrane protein Smoothened is released, causing the transport of the transcription factor glioma-associated oncogene (Gli) into the nucleus, where it regulates the transcription of target genes that control cell differentiation, survival and growth $(5,6)$. Three Gli genes (Gli1, Gli2 and Gli3) have been identified in mammalian tissues, among which Gli1 is considered to be the only loyal marker of hedgehog pathway activity (8-10). Several recent 
studies have shown that the hedgehog pathway is associated with the migration and invasion of ovarian, pancreatic, esophageal and gastric carcinomas (6,11-13). However, to date, the implication of the hedgehog pathway in the aggressiveness of GBM has not been elucidated. Furthermore, the mechanisms by which the hedgehog signaling pathway is involved in GBM migration and invasion require further elucidation.

Matrix metalloproteinase-2 (MMP-2) and MMP-9, zinc-dependent endopeptidases, are able to degrade nearly all extracellular matrix components to promote cancer-cell invasion (14). It has been demonstrated that MMP-2 and MMP-9 are mostly associated with migration and invasion in multiple tumor types (14-16). Further studies have shown that the expression of MMP-2 and MMP-9 in several cancer types is mediated by numerous signaling pathways, which include the hedgehog signaling pathway, the wnt/ $\beta$-catenin pathway, the phosphoinositide-3 kinase (PI3K)/AKT pathway and the mitogen-activated protein kinase (MAPK) pathway $(10,17,18)$. It has been suggested that the hedgehog signaling pathway is cross-linked with other signaling pathways. However, in GBM, it has remained elusive whether there is a link between the hedgehog signaling pathway and the PI3K/AKT pathway or whether the hedgehog signaling pathway regulates the expression of MMP-2 and MMP-9 via the PI3K/AKT.

The present study examined the role of the hedgehog pathway in the migration and invasion of GBM, specifically focusing on the link between the hedgehog signaling pathway and the PI3K/AKT signaling pathway. Specifically, the present study assessed whether the hedgehog pathway is involved in human GBM migration and invasion by induction of MMP-2 and MMP-9 via the PI3K/AKT signaling pathway.

\section{Materials and methods}

Human samples. Fifty-one patients with GBM who underwent surgery at the Second Affiliated Hospital of Harbin Medical University (Harbin, China) between March 2010 and July 2013 were enrolled in the present study. All tumor specimens were pathologically diagnosed as GBM. Written informed consent was given by the patients for their information to be stored in the hospital database and used for research. Approval was obtained from the Research Ethics Committee of the Second Affiliated Hospital of Harbin Medical University (Harbin, China).

Reagents. Dulbecco's modified Eagle's medium [(DMEM)/F12] was obtained from GE Healthcare Life Sciences (Logan, UT, USA). Mouse anti-MMP-2 monoclonal antibody (cat. no. SC-13595) and mouse MMP-9 monoclonal antibody (cat. no. SC-21733) were purchased from Santa Cruz Biotechnology, Inc. (Dallas, TX, USA). Rabbit monoclonal phospho-AKT ${ }^{\mathrm{S} 473}$ antibody (cat. no. \#4060) and mouse AKT monoclonal antibody (cat. no. \#2920) were purchased from Cell Signaling Technology, Inc. (Danvers, MA, USA). Rabbit polyclonal $\beta$-actin antibody (cat. no. BS-0061R) was purchased from Bioss (Beijing, China). Biotin-labelled goat anti-rabbit secondary antibody (cat. no. ZDR-5306) and biotin-labelled goat anti-mouse secondary antibody (cat. no. ZDR-5307) were purchased from ZSJB-BIO (Beijing, China). Recombinant human sonic hedgehog N-terminal peptide (rhSHH; cat. no. 1314-SH) was purchased from Toronto Research Chemicals (Toronto, Canada). Cyclopamine (cat. no. BML-GR334) and GM6001 (cat. no. BML-EI300), were obtained from Enzo Life Sciences, Inc. (Farmingdale, NY, USA). Ly294002 (cat. no. L9908) was from purchased from Sigma-Aldrich (St. Louis, MO, USA)

Cell lines and culture conditions. The human glioma U251 cell line, purchased from the Chinese Academy of Science Cell Bank (Shanghai, China), was grown in DMEM/F12 supplemented with $100 \mathrm{U} / \mathrm{ml}$ penicillin (Beyotime Institute of Biotechnology, Haimen, China), $100 \mathrm{mg} / \mathrm{ml}$ streptomycin (Beyotime Institute of Biotechnology) and 10\% fetal bovine serum (FBS; GE Healthcare Life Sciences) in a humidified incubator $\left(5 \% \mathrm{CO}_{2}\right.$ and $\left.37^{\circ} \mathrm{C}\right)$.

Cell adhesion assay. The U251 cells were incubated for $24 \mathrm{~h}$ with various concentrations of cyclopamine $(0,5.0$ and $10.0 \mu \mathrm{m})$ or $\mathrm{rhSHH}(0,0.5$ and $1.0 \mu \mathrm{g} / \mathrm{ml})$. The 96 -well plates were coated with $5 \mathrm{mg} / \mathrm{ml}$ fibronectin (Sigma-Aldrich) and blocked with $1 \%$ bovine serum albumin (ZSGB-BIO, Beijing, China) for $4 \mathrm{~h}$. U251 cells $(20,000$ cells/well) were then seeded into the 96 -well plates and incubated for $1 \mathrm{~h}$ at $37^{\circ} \mathrm{C}$ in $5 \%$ $\mathrm{CO}_{2}$. Subsequently, the medium was gently removed by aspiration and the wells were washed twice with phosphate-buffered saline (PBS) to remove any non-adherent cells. The adherent cells were quantified using an MTT assay, as we previously described (19).

Migration and invasion assay. The U251 cells were either untreated or pre-treated with GM6001 (15 $\mu \mathrm{m})$, anti-MMP-2 $(20 \mu \mathrm{g} / \mathrm{ml})$ or anti-MMP-9 neutralizing antibodies $(20 \mu \mathrm{g} / \mathrm{ml})$ for $1 \mathrm{~h}$. The cell invasion assay was performed using a 24-well Transwell chamber (cat no. 3422; Corning-Costar Inc., Corning, NY, USA) with a pore size of $8 \mu \mathrm{m}$. Polycarbonate filters were coated with $20 \mu$ l diluted Matrigel (1:1 in DMEM/F12; Corning Incorporated, Corning, NY, USA) on the upper side. $1 \times 10^{5}$ cells/well were suspended in $0.2 \mathrm{ml}$ serum-free medium with various concentrations of cyclopamine $(0,5.0$ and $10.0 \mu \mathrm{m})$ or $\operatorname{rhSHH}(0,0.5$ and $1.0 \mu \mathrm{g} / \mathrm{ml})$, and then seeded in the upper chamber, while medium $(0.5 \mathrm{ml})$ containing $10 \% \mathrm{FBS}$ as a chemoattractant was added to the lower chamber. After a $24-\mathrm{h}$ incubation period at $37^{\circ} \mathrm{C}$ with $5 \% \mathrm{CO}_{2}$, the non-invaded cells and the Matrigel on the upper surface of the filter were removed using a cotton swab and cells on the lower surface of the filter were fixed with methanol (Beyotime Institute of Biotechnology) for $10 \mathrm{~min}$, stained with hematoxylin (ZSGB-BIO, Beijing, China) for $2 \mathrm{~min}$, rinsed in running tap water for $10 \mathrm{~min}$, differentiated with $0.3 \%$ acid alcohol (ZSGB-BIO) for $1 \mathrm{~min}$, rinsed in running tap water for $10 \mathrm{~min}$, and finally stained with eosin (ZSGB-BIO) for $2 \mathrm{~min}$. The migration assay was performed as described in the invasion assay but with a shorter incubation period (12 h) and no matrigel coating. A Leica DM2700 M light microscope (Leica Microsystems GmbH, Wetzlar, Germany) was used for evaluating cell number. The cell number on the lower surface of the filter was evaluated by counting three random microscopic fields (magnification, $\mathrm{x} 400$ ) and the numbers of migrated or invaded cells were averaged. 
RNA extraction and reverse transcription quantitative polymerase chain reaction analysis. The $\mathrm{U} 251$ cells were incubated for $24 \mathrm{~h}$ with various concentrations of cyclopamine $(0,5.0$ and $10.0 \mu \mathrm{m})$ or rhSHH $(0,0.5$ and $1.0 \mu \mathrm{g} / \mathrm{ml})$, and then incubated with or without Ly294002 $(25 \mu \mathrm{M})$ for $1 \mathrm{~h}$. Total RNA was isolated from cells using TRIzol (Invitrogen Life Technologies, Inc., Carlsbad, CA, USA) according to the manufacturer's instructions. $1 \mu \mathrm{g}$ RNA was reversely transcribed into cDNA using a High-Capacity cDNA reverse transcription kit (Applied Biosystems, Thermo Fisher Scientific, Waltham, MA, USA). qPCR was performed using SYBR Green PCR Master Mix (Applied Biosystems) in a total volume of $20 \mu \mathrm{l}$ on an ABI Prism 7500 sequence detection system (Applied Biosystems). GAPDH, MMP-2 and MMP-9 primers were as follows: MMP-2 sense, 5'-GGTTGTCTGAAGTCACTGCACAGT-3' and anti-sense, 5'-CTCGGTAGGGACATGCTAAGTAGAG-3'; MMP-9 sense, 5'-GCTGGGCTTAGATCATTCCTCA-3' and anti-sense, 5'-AGGGCGAGGACCATAGAGGT-3'; GAPDH sense, 5'-CCTCCCGCTTCGCTCTCT-3' and anti-sense, 5'-CTGGCGACGCAAAAGAAGA-3'. All primers were synthesized by the Invitrogen Life Technologies. The reaction conditions were as follows: $94^{\circ} \mathrm{C}$ for $5 \mathrm{~min}, 35$ cycles at $94^{\circ} \mathrm{C}$ for $30 \mathrm{sec}, 55^{\circ} \mathrm{C}$ for $30 \mathrm{sec}, 72^{\circ} \mathrm{C}$ for $2 \mathrm{~min}$ and a final extension at $72^{\circ} \mathrm{C}$ for $5 \mathrm{~min}$. The mRNA expression levels were normalized to GAPDH using the standard $\Delta \Delta \mathrm{Ct}$ method.

Western blot analysis. The U251 cells were incubated for $24 \mathrm{~h}$ with various concentrations of cyclopamine $(0,5.0$ and $10.0 \mu \mathrm{m})$ or $\mathrm{rhSHH}(0,0.5$ and $1.0 \mu \mathrm{g} / \mathrm{ml})$, and then incubated with or without Ly294002 $(25 \mu \mathrm{M})$ for $1 \mathrm{~h}$. Cells were washed with PBS and total protein was extracted from cells with lysis buffer [50 mmol/1 Tris-HCl ( $\mathrm{pH} \mathrm{7.4;} \mathrm{Sigma-Aldrich,}$ St. Louis, MO, USA), $150 \mathrm{mmol} / 1 \mathrm{NaCl}$ (Beyotime Institute of Biotechnology), 0.5\% NP-40 (Sigma-Aldrich), $1 \mathrm{mmol} / 1$ EDTA (Sigma-Aldrich), $25 \mathrm{mmol} / 1 \mathrm{NaFl}$ (Beyotime Institute of Biotechnology), $10 \mathrm{mmol} / 1 \mathrm{Na} 3 \mathrm{VO} 4$ (Sigma-Aldrich) and $1 \mathrm{mmol} / \mathrm{l}$ phenylmethanesulfonylfluoride $(\mathrm{pH} \mathrm{7.4;}$ Sigma-Aldrich)] on ice for $15 \mathrm{~min}$. After centrifugation, the bicinchoninic acid protein assay kit (Beyotime Institute of Biotechnology) was used to determine the protein concentration. Equal amounts of $80 \mu \mathrm{g}$ protein were loaded onto $15 \%$ SDS-PAGE gels and transferred to $0.45-\mu \mathrm{m}$ polyvinylidene difluoride membranes (Millipore, Billerica, MA, USA). After blocking with 5\% non-fat milk for $1 \mathrm{~h}$, the membranes were incubated overnight at $4^{\circ} \mathrm{C}$ with antibody against phospho-AKT ${ }^{\mathrm{S} 473}(1: 1,000)$, AKT $(1: 1,000), \beta$-actin $(1: 1,000)$, MMP-2 (1:500) or MMP-9 (1:500). After three washes with PBS containing Tween 20 for $5 \mathrm{~min}$, the membranes were incubated with horseradish peroxidase-conjugated secondary antibodies against rabbit $(1: 2,000)$ or mouse $(1: 2,000)$ for $1 \mathrm{~h}$ at room temperature. Protein bands were detected by enhanced chemiluminescence (cat. no. PK-MB902-500-500; Thermo Fisher Scientific) and quantified by Quality One Bio-Rad Gel Imaging 4.52 system (Bio-Rad Laboratories, Inc., Hercules, CA, USA).

Immunohistochemical staining. Formalin-fixed and paraffin-embedded GBM tissues were sliced into $4-\mu \mathrm{m}$ sections. The tumor sections were then dewaxed, re-hydrated and immersed in $3 \% \mathrm{H}_{2} \mathrm{O}_{2}$ (ZSGB-BIO). Antigen retrieval was achieved by heating the sections at $95^{\circ} \mathrm{C}$ for $10 \mathrm{~min}$ in $0.01 \mathrm{~mol} / 1$ citrate buffer ( $\mathrm{pH}$ 6.0; ZSGB-BIO). To reduce nonspecific reactivity, the sections were treated with $10 \%$ normal goat serum (ZSGB-BIO) for $60 \mathrm{~min}$. Subsequently, the sections were incubated overnight at $4^{\circ} \mathrm{C}$ with primary antibodies against phospho-AKT ${ }^{\mathrm{S} 473}$ (1:50), Gli1 (1:50; Abcam, Cambridge, UK), MMP-2 (1:100) and MMP-9 (1:100). Following three washes with PBS for 5 min and incubation with secondary antibody (anti-rabbit/anti-mouse; ZSGB-BIO) for $1 \mathrm{~h}$ at room temperature, antibodies were visualized done using a 3,3'-diaminobenzidine substrate kit (cat. no. ZLI-9017; ZSGB-BIO). Immunohistochemical analysis was performed as previously described using a Leica DM2700M light microscope (20).

Statistical analysis. Values are expressed as the mean \pm standard deviation. SPSS 19.0 statistical software (International Business Machines, Armonk, NY, USA) was used for statistical analysis. Statistical significance was determined by Student's $t$-test or Spearman's correlation test. $\mathrm{P}<0.05$ was considered to indicate a statistically significant difference between values.

\section{Results}

Inhibition of hedgehog signaling inhibits the adhesion, invasion and migration of GBM cells. Cyclopamine, a specific antagonist of the hedgehog pathway, was used to evaluate the effects of the hedgehog pathway on the adhesion, invasion and migration of U251 cells. The results showed that cyclopamine suppressed the adhesion of U251 cells in a dose-dependent manner (Fig. 1A). Furthermore, Transwell migration and invasion assays were performed following treatment of the cells with $0,5.0$ or $10.0 \mu \mathrm{M}$ cyclopamine for $12 \mathrm{~h}$ (cell migration) and $24 \mathrm{~h}$ (cell invasion), respectively. As demonstrated in Fig. 1B and C, cyclopamine significantly reduced U251-cell migration and invasion in a dose-dependent manner. These results demonstrated that inactivation of the hedgehog pathway markedly inhibited the adhesion, invasion and migration of U251 cells.

Enhancement of hedgehog signaling enhances the adhesion, invasion and migration of GBM cells. To determine the effects of hedgehog signaling on cell attachment, U251 cells were treated with rhSHH and their adhesion ability was evaluated. As illustrated in Fig. 2A, rhSHH dose-dependently increased the adhesion ability of U251 cells. Furthermore, Transwell migration and invasion assays were performed following treatment of the cells with $0,0.5$ or $1.0 \mu \mathrm{g} / \mathrm{ml} \mathrm{rhSHH}$ for $12 \mathrm{~h}$ (cell migration) or $24 \mathrm{~h}$ (cell invasion), respectively. As shown in Fig. 2B and $\mathrm{C}$, the number of migrated or invaded cells was increased by rhSHH in a dose-dependent manner. These results demonstrated that activation of the hedgehog pathway markedly increased the adhesion, invasion and migration of U251 cells.

Hedgehog signaling enhances cell migration and invasion by increasing the expression of MMP-2 and MMP-9 in GBM. It is known that MMP-2 and MMP-9 have important roles in tumor migration and invasion (14-16). Therefore, the present study investigated whether the hedgehog pathway regulated the 

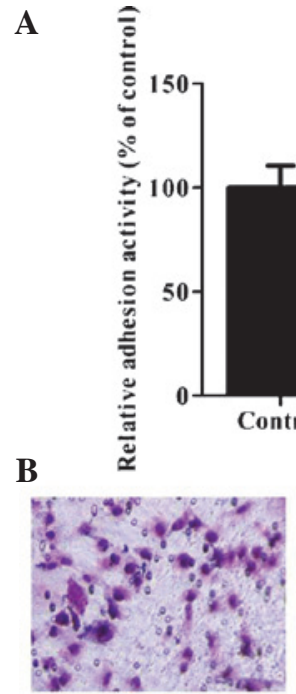

o

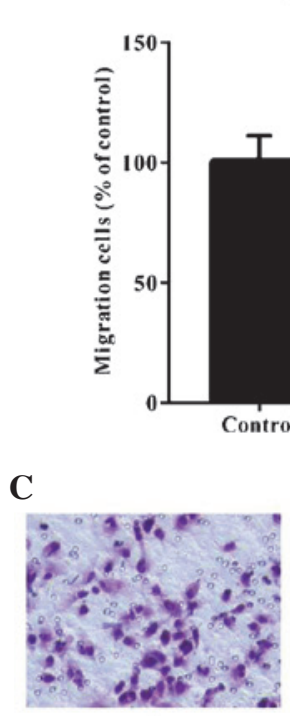

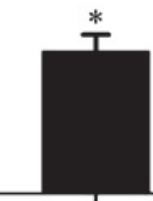
Cyclopamine $(\mu \mathrm{M})$

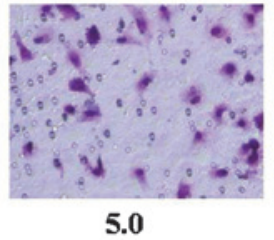

Cyclopamine $(\mu \mathrm{M})$

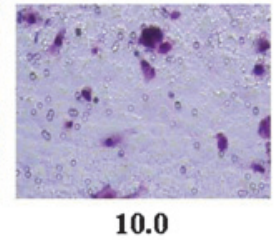

10.0
C
0

$\mathbf{0}$

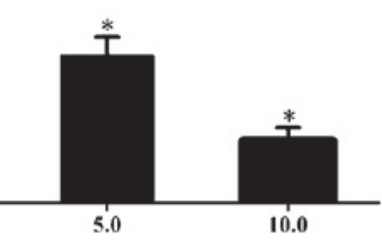

Cyclopamine $(\mu \mathrm{M})$

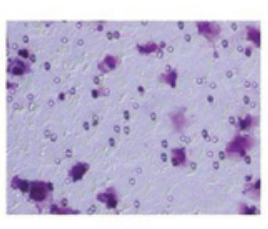

5.0

Cyclopamine $(\mu \mathrm{M})$

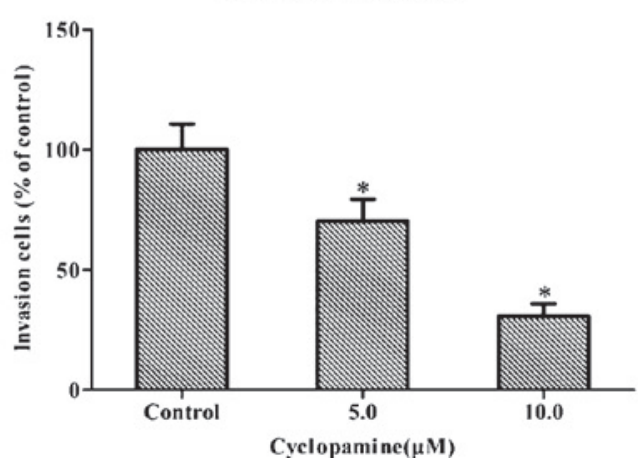

Figure 1. Cyclopamine inhibits adhesion, invasion and migration of U251 cells (A) U251 cells were incubated for $24 \mathrm{~h}$ with various concentrations of cyclopamine. Cells were seeded into 96-well plates coated with fibronectin. After $1 \mathrm{~h}$, the adhered cells were analyzed by MTT assay. The adhesion rate was expressed as a percentage of the control $(0 \mu \mathrm{m})$. (B) U251 cells were seeded in the upper wells without matrigel coating and treated with various concentrations of cyclopamine. After $12 \mathrm{~h}$, cells on the bottom side of the filter were fixed, stained and counted (magnification, $x 400$ ). The migration rate was expressed as a percentage of the control $(0 \mu \mathrm{m})$. (C) U251 cells were seeded in the matrigel-coated upper wells and treated with various concentrations of cyclopamine. After $24 \mathrm{~h}$, cells on the bottom side of the filter were fixed, stained and counted (magnification, $\mathrm{x} 400$ ). The invasion rate was expressed as a percentage of the control $(0 \mu \mathrm{m})$. Values are expressed as mean \pm standard deviation of three independent experiments. ${ }^{*} \mathrm{P}<0.001$, compared with controls.
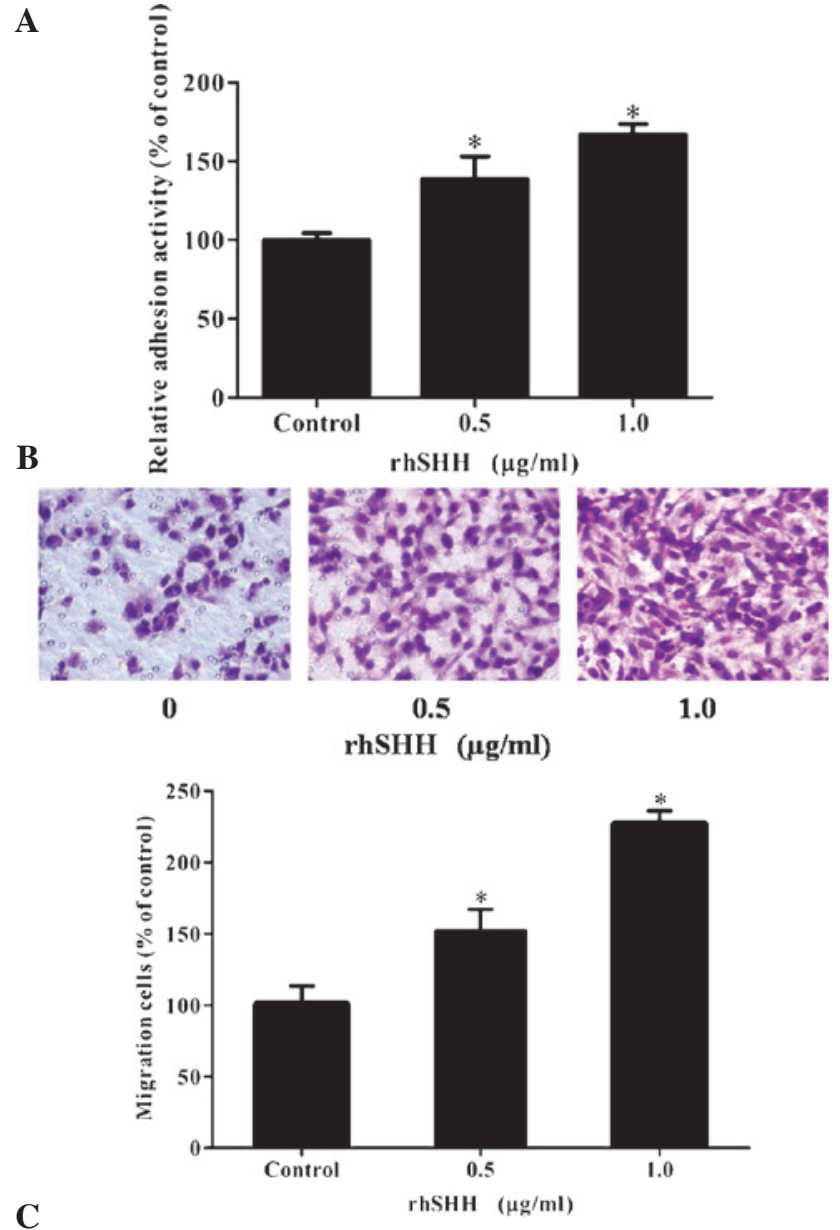

C
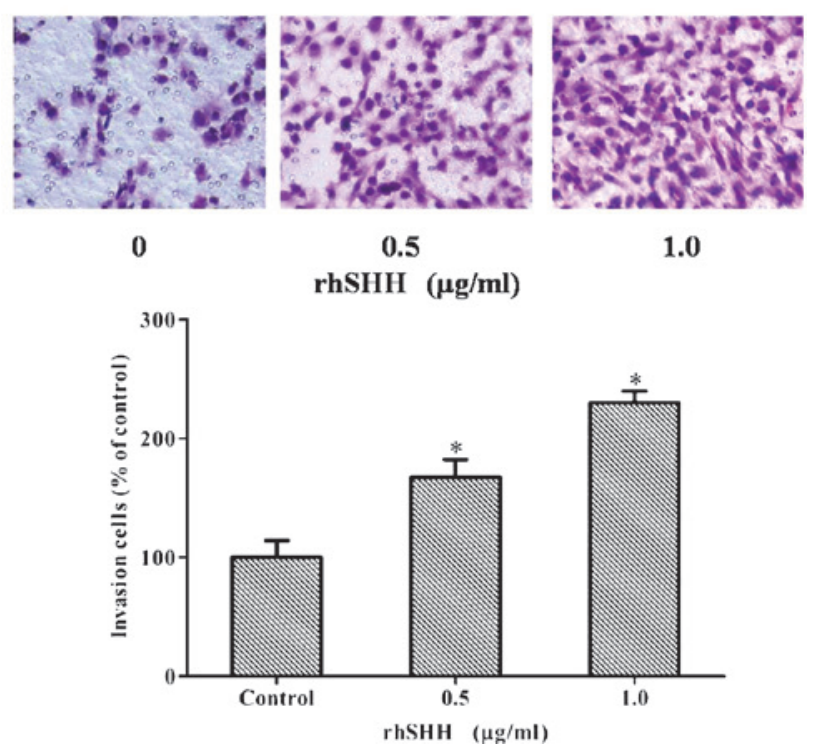

Figure 2.rhSHH enhances the adhesion, invasion and migration of U251 cells (A) $\mathrm{U} 251$ cells were incubated for $24 \mathrm{~h}$ with various concentrations of $\mathrm{rhSHH}$. Cells were seeded into 96-well plates coated with fibronectin. After $1 \mathrm{~h}$, the adhered cells were analyzed by MTT assay. The adhesion rate was expressed as a percentage of the control $(0 \mu \mathrm{g} / \mathrm{ml})$. (B) $\mathrm{U} 251$ cells were seeded in the upper wells without matrigel coating and treated with various concentrations of rhSHH. After $12 \mathrm{~h}$, cells on the bottom side of the filter were fixed, stained and counted (magnification, $\mathrm{x} 400$ ). The migration rate was expressed as a percentage of the control $(0 \mu \mathrm{g} / \mathrm{ml})$. (C) U251 cells were seeded in the upper wells with matrigel coating and treated with various concentrations of rhSHH. After $24 \mathrm{~h}$, cells on the bottom side of the filter were fixed, stained and counted (magnification, $\mathrm{x} 400$ ). The invasion rate was expressed as a percentage of the control $(0 \mu \mathrm{g} / \mathrm{ml})$. Values are expressed as the mean \pm standard deviation of three independent experiments. ${ }^{*} \mathrm{P}<0.001$, compared with controls. rhSHH, recombinant human sonic hedgehog $\mathrm{N}$-terminal peptide. 


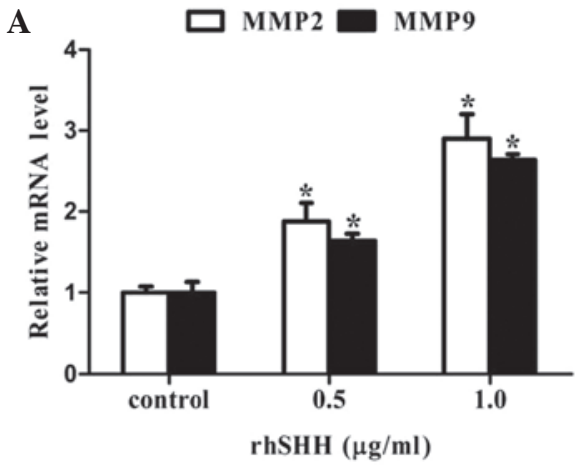

C
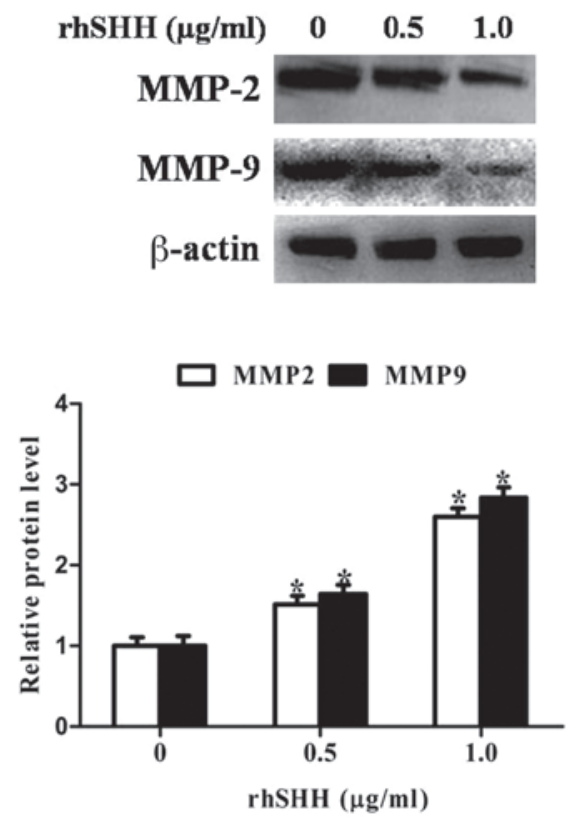

$\mathbf{E}$

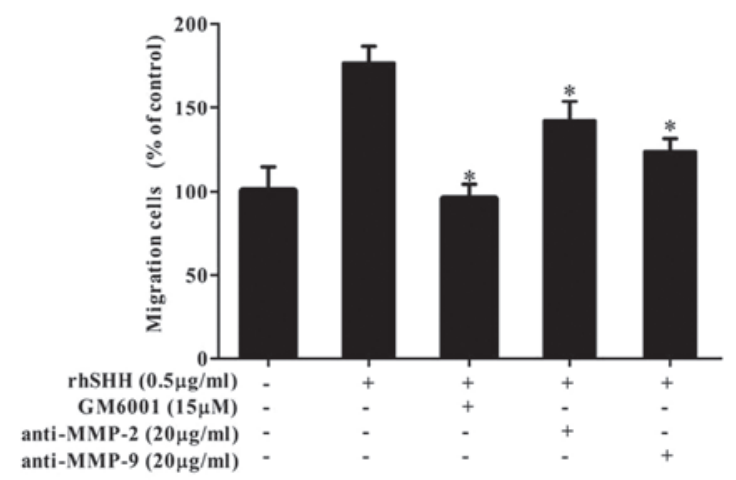

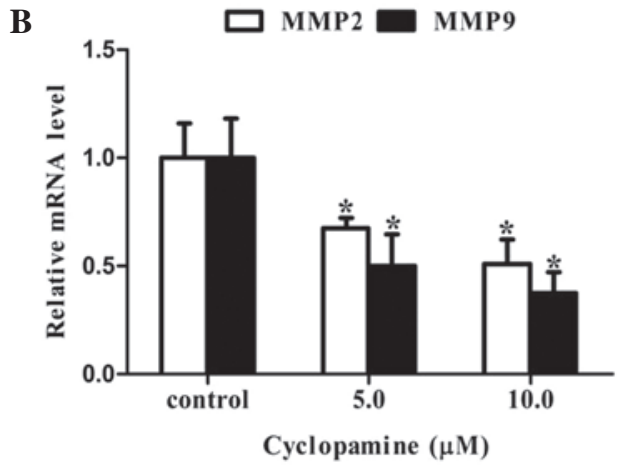

D
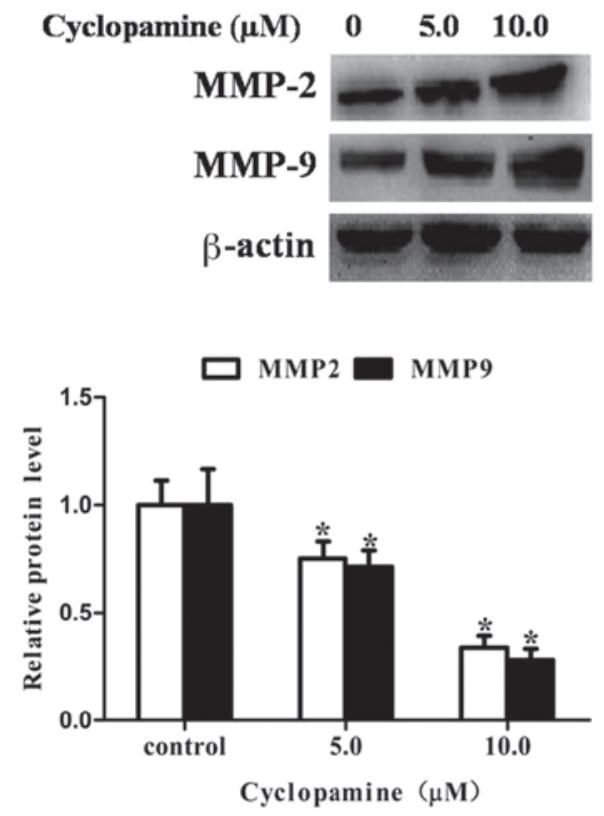

$\mathbf{F}$

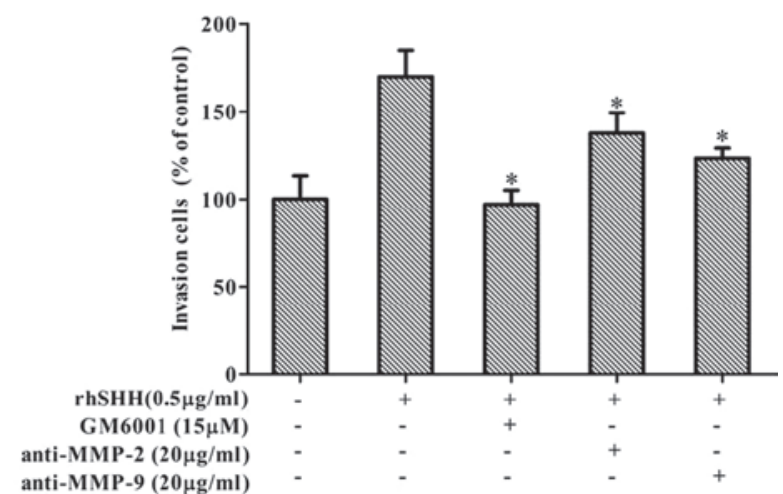

Figure 3. Hedgehog signaling affects cell migration and invasion by regulating the expression of MMP-2 and MMP-9 in glioblastoma cells. (A and B) U251 cells were incubated for $24 \mathrm{~h}$ with various concentrations of rhSHH or cyclopamine for $24 \mathrm{~h}$. The mRNA levels of MMP-2 and MMP-9 were assayed by reverse transcription quantitative polymerase chain reaction and normalized to GAPDH. (C and D) U251 cells were incubated with various concentrations of rhSHH or cyclopamine for $24 \mathrm{~h}$. The protein levels of MMP-2 and MMP-9 in cell lysates were analyzed by western blot, and band intensity was quantified by densitometry and normalized to $\beta$-actin. (E and F) U251 cells were incubated for $24 \mathrm{~h}$ with $0.5 \mu \mathrm{g} / \mathrm{ml} \mathrm{rhSHH}$ or left untreated (control). U251 cells were either left untreated (control) or pre-treated with GM6001, anti-MMP-2 or MMP-9 anti-MMP-9 neutralizing antibodies for 1 h. Subsequently, Transwell migration and invasion assays were performed. Results are presented as percentages of the control. Values are expressed as the mean \pm standard deviation of three independent experiments. " $\mathrm{P}<0.001$, compared with controls. MMP, matrix metalloproteinase; rhSSH, recombinant human sonic hedgehog $\mathrm{N}$-terminal peptide.

expression of MMP-2 and MMP-9. It was observed that treatment with rhSHH dose-dependently upregulated the mRNA levels of MMP-2 and MMP-9 in U251 cells (Fig. 3A), while cyclopamine treatment decreased the mRNA levels of MMP-2 and MMP-9 in U251 cells in a concentration-dependent manner (Fig. 3B). Similarly, rhSHH dose-dependently upregulated the protein levels of MMP-2 and MMP-9 in U251 cells (Fig. 3C), while cyclopamine treatment decreased the protein levels of 
A

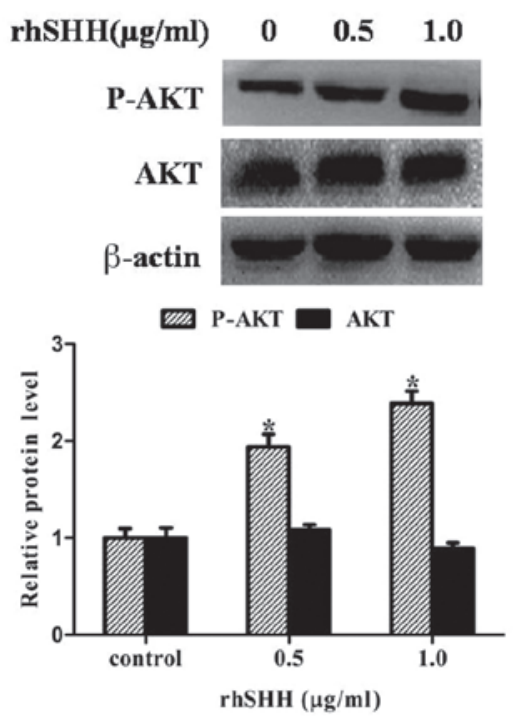

B

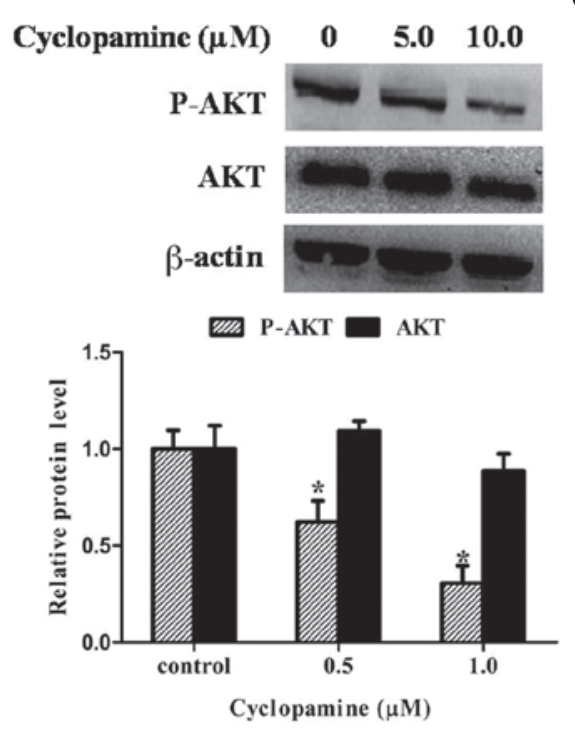

C $\begin{array}{rrrr}\operatorname{rhSHH}(0.5 \mu \mathrm{g} / \mathrm{ml}) & - & + & + \\ \operatorname{LY90042}(25 \mathrm{mM}) & - & - & +\end{array}$

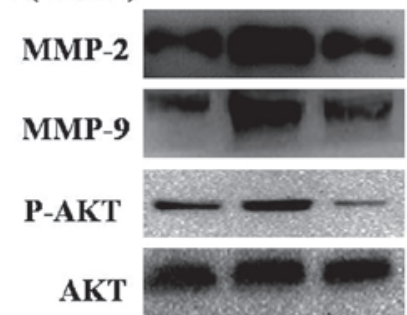

$\beta$-actin
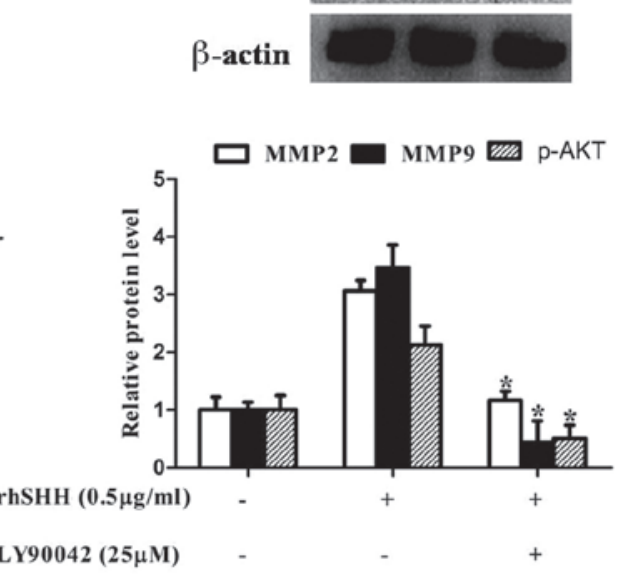

LY90042 $(25 \mu \mathrm{M})$

D

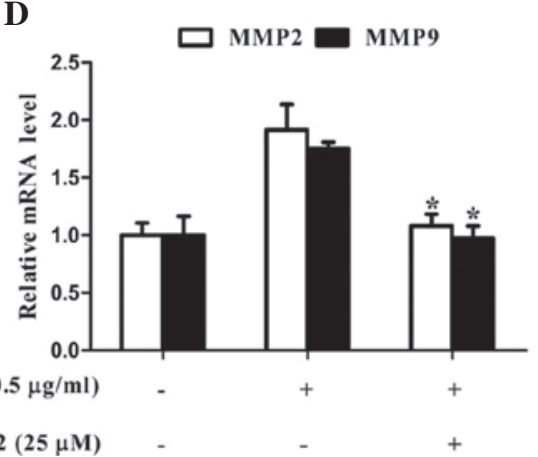

LY90042(25

Figure 4. Hedgehog signaling regulates the expression of MMP-2 and MMP-9 through the phosphoinositide-3 kinase/AKT pathway in glioblastoma cells. (A and B) U251 cells were incubated with various concentrations of rhSHH or cyclopamine for $24 \mathrm{~h}$. The protein levels of p-AKT and AKT in cell lysates were analyzed by western blot and band intensities were quantified by densitometry and normalized to $\beta$-actin. * P<0.001, compared with controls. (C) U251 cells were treated with or without rhSHH $(0.5 \mu \mathrm{g} / \mathrm{ml})$ for $24 \mathrm{~h}$ and then incubated with or without Ly $294002(25 \mu \mathrm{M})$ for $1 \mathrm{~h}$. The protein levels of MMP-2, MMP-9, p-AKT and AKT in cell lysates were analyzed by western blot. The band intensity of MMP-2, MMP-9 and p-AKT was quantified by densitometry and normalized to $\beta$-actin. (D) U251 cells were treated with or without rhSHH $(0.5 \mu \mathrm{g} / \mathrm{ml})$ for $24 \mathrm{~h}$ and then incubated with or without Ly $294002(25 \mu \mathrm{M})$ for $1 \mathrm{~h}$. The mRNA levels of MMP-2 and MMP-9 were assayed by reverse transcription quantitative polymerase chain reaction. ${ }^{*} \mathrm{P}<0.05$, compared with rhSHH-treated group. Values are expressed as the mean \pm standard deviation of three independent experiments. MMP, matrix metalloproteinase; rhSSH, recombinant human sonic hedgehog N-terminal peptide; p, phosphorylated.

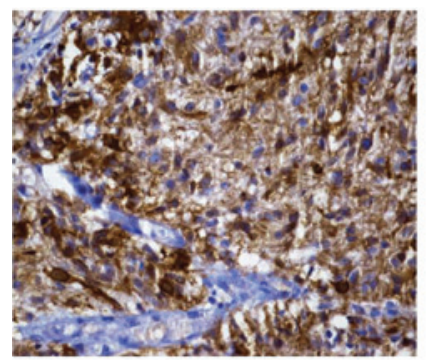

Gli1

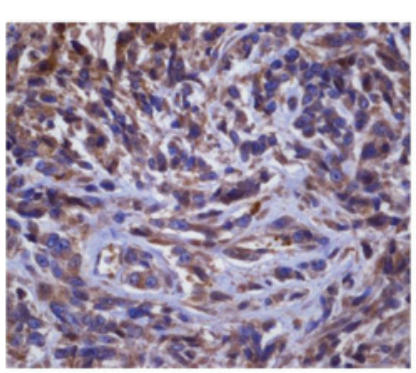

phospho-AKT

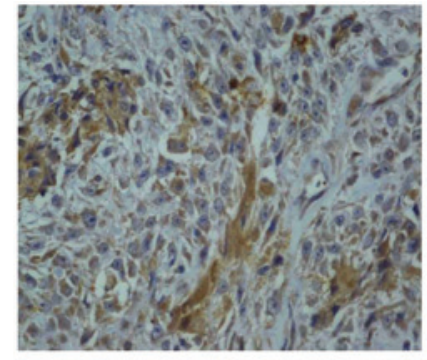

MMP-2

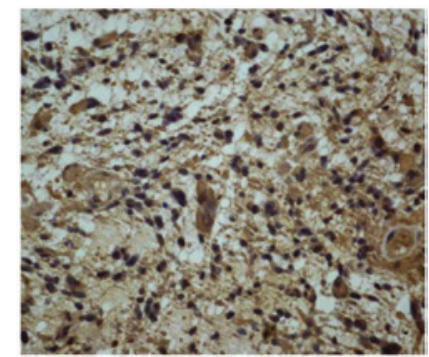

MMP-9

Figure 5. Representative images of immunohistochemical staining of 51 glioblastoma tissues for Gli1, phospho-AKT, MMP-2 and MMP-9 (magnification, x400). Images show positive expression of Gli1, phospho-AKT, MMP-2 and MMP-9. Expression of MMP-2 and MMP-9 was defined as cytoplasmic staining. Gli1 and phospho-AKT were located in cytoplasm and nuclei. MMP, matrix metalloproteinase; Gli1, glioblastoma-associated oncogene 1.

MMP-2 and MMP-9 in U251 cells in a concentration-dependent manner (Fig. 3D). Furthermore, MMP-2 neutralizing antibody (anti-MMP-2), MMP-9 neutralizing antibody (anti-MMP-9) or GAM6001 were able to inhibit the rhSHH-induced cell migration and invasion (Fig. 3E and F). These results demonstrated that hedgehog signaling enhanced the expression of MMP-2 and MMP-9 to promote cell migration and invasion of GBM.

Hedgehog signaling regulates the expression of $M M P-2$ and MMP-9 through the PI3K/AKT pathway in GBM cells. In 
Table I. Correlation between Gli1, phospho-AKT, MMP-2 and MMP-9 protein expression.

\begin{tabular}{lcccr}
\hline & \multicolumn{2}{c}{ Gli1 expression } & & \\
\cline { 2 - 3 } Variable & Negative (n) & Positive (n) & P-value & r \\
\hline Phospho-AKT expression & 13 & 7 & $<0.001$ & 0.582 \\
$\quad$ Negative & 3 & 28 & & 0.380 \\
$\quad$ Positive & & & & 0.013 \\
MMP-2 expression & 8 & 5 & & \\
$\quad$ Negative & 8 & 30 & & 0.019 \\
$\quad$ Positive & 9 & 8 & & \\
MMP-9 expression & 7 & 27 & & \\
$\quad$ Negative & & & & \\
$\quad$ Positive &
\end{tabular}

MMP, matrix metalloproteinase; Gli1, glioma-associated oncogene 1.

human glioma cells, the PI3K/Akt pathway is one of the major signaling pathways for the invasive process. Therefore, the present study determined whether activation of the hedgehog pathway is associated with alterations of the PI3K/Akt pathway in GBM. Fig. 4A and B shows that AKT phosphorylation was significantly enhanced by $\mathrm{rhSHH}$, while it was significantly inhibited by cyclopamine. However, the total protein expression of AKT was not altered by rhSHH and cyclopamine. To confirm whether MMP-2 and MMP-9 expression, mediated by the hedgehog pathway, was associated with the PI3K/AKT pathway in GBM cells, this pathway was blocked using PI3K inhibitor Ly294002. Fig. 4C and D shows that Ly294002 significantly decreased rhSHH-induced mRNA and protein expression of MMP-2 and MMP-9 as well as phosphorylation of AKT. These results revealed that hedgehog signaling regulated the expression of MMP-2 and MMP-9 through the $\mathrm{PI} 3 \mathrm{~K} / \mathrm{AKT}$ pathway in GBM cells.

Glil expression is correlated with phospho-AKT, MMP-2 and $M M P-9$ expression in GBM. The present study further examined the correlation between Gli1, phospho-AKT, MMP-2 and MMP-9 expression in GBM. The expression of Gli1, phospho-AKT, MMP-2 and MMP-9 in the 51 GBM samples was evaluated using immunohistochemical analysis (Fig. 5). As shown in Table I, Gli1 protein expression was positively correlated with the expression of phospho-AKT $(\mathrm{r}=0.582$; $\mathrm{P}<0.001)$, MMP-2 ( $\mathrm{r}=0.380 ; \mathrm{P}=0.013)$ and MMP-9 $(\mathrm{r}=0.329$; $\mathrm{P}=0.019)$. These results further indicated that the expression of MMP-2 and MMP-9 is mediated by the hedgehog signaling pathway via PI3K/AKT signaling.

\section{Discussion}

Glioblastoma is the most common type of malignant tumor of the central nervous system. Migration and invasion are the leading reasons for treatment failure and cancer-associated mortality of GBM patients $(3,4)$. Therefore, it is necessary to develop novel prevention and treatment strategies for GBM, for which an enhanced knowledge of the underlying molecular mechanisms of migration and invasion of GBM is required. The present study posed and verified the hypothesis that the hedgehog signaling pathway activates the PI3K/AKT pathway, which subsequently upregulates the expression of MMP-2 and MMP-9 at the mRNA and protein level, thereby mediating GBM migration and invasion.

The hedgehog signaling pathway has a vital role in the control of cell differentiation, cell proliferation and embryonic development $(5,7,12)$. According to recent studies, aberrant activation of hedgehog signaling is linked to the aggressiveness of multiple tumor types, including $\operatorname{GBM}(21,22)$. The results of the present study are therefore consistent with those of previous studies. The present study demonstrated that activation of hedgehog signaling by rhSHH promoted the adhesion, invasion and migration of GBM cells, whereas inhibition of this signaling with cyclopamine, an antagonist of the hedgehog pathway, suppressed the adhesion, invasion and migration of GBM cells. These results suggested that the hedgehog signaling pathway has a tumor-promoting role in GBM that regulates cell invasion and migration.

MMP-2 and MMP-9 are important members of the MMP family, which and promote cancer-cell migration and invasion by degrading extracellular matrix components (2,14-17). An increasing number of studies demonstrated that MMP-2 and MMP-9 have important roles in GBM angiogenesis and can be regulated by multiple pathways (23-25). The present study examined MMP-2 and MMP-9 expression to explore the association between the hedgehog pathway and cell invasion and migration. It was found that rhSHH dose-dependently upregulated mRNA and protein levels of MMP-2 and MMP-9 in U251 cells, whereas cyclopamine decreased mRNA and protein levels of MMP-2 and MMP-9 in U251 cells in a concentration-dependent manner. In addition, U251 cells were treated with MMP-2- or MMP-9-neutralizing antibody or with GAM6001, and their effects on rhSHH-induced migration and invasion of GBM cells was evaluated. A Transwell assay revealed that MMP-2- and MMP-9 neutralizing antibody or GAM6001 were able to reverse rhSHH-induced cell migration and invasion. These results indicated that hedgehog signaling 
promotes cell migration and invasion by enhancing the expressions of MMP-2 and MMP-9 in GBM cells.

Accumulating evidence revealed that the PI3K/Akt pathway is activated in multiple types of cancer, including kidney, lung, liver and ovarian cancer, and has an important role in migration and invasion (26-28). Several studies reported that the PI3K/Akt pathway is mediated by the SHH pathway. For example, Yoo et al (29) reported that hedgehog signaling promotes the metastasis of gastric cancer through activation of the PI3K/Akt pathway. Morton et al (30) also demonstrated that inhibition of the hedgehog pathway markedly regulated pancreatic tumorigenesis through inhibition of the PI3K/AKT pathway. However, it remains elusive whether such association also exists in GBM. In the present study, AKT phosphorylation of GBM cells was significantly enhanced by rhSHH and was significantly inhibited by cyclopamine, verifying the hypothesis that the PI3K/Akt pathway is mediated via the hedgehog pathway in GBM cells. Furthermore, the present study demonstrated that the PI3K inhibitor Ly294002 significantly decreased rhSHH-induced MMP-2 and MMP-9 mRNA and protein expression as well as phosphorylation of AKT. More importantly, immunohistochemical analysis showed that Gli1 protein expression was closely correlated with phospho-AKT, MMP-2 and MMP-9 in GBM samples. All of these results suggested that hedgehog signaling regulates the expression of MMP-2 and MMP-9 through the PI3K/AKT pathway in GBM.

In conclusion, the present study demonstrated for the first time, to the best of our knowledge, that the hedgehog signaling pathway promotes the invasion and migration of GBM cells by enhancing MMP-2 and MMP-9 expression via the PI3K/AKT pathway. These results provided a novel molecular mechanism of GBM-cell aggressiveness based on the hedgehog signaling pathway and may assist in the discovery of novel therapeutic targets to control the aggressiveness of GBM.

\section{Acknowledgements}

The present study was supported by the National High Technology Research and Development Program of China (863 program; grant no. 2012AA02A508), the National Basic Research Program of China (973 program; grant no. 2012CB517803), the National Natural Science Foundation of China (grant nos. 81070217 and 81270340) and the Research Foundation of the Chinese Ministry of Health (grant no. w2011bx059).

\section{References}

1. Louis DN, Ohgaki H, Wiestler OD, Cavenee WK, Burger PC, Jouvet A, Scheithauer BW and Kleihues P: The 2007 WHO classification of tumours of the central nervous system. Acta Neuropathol 114: 97-109, 2007.

2. DeAngelis LM: Brain tumors. N Engl J Med 344: 114-123, 2001.

3. Matsumura H, Ohnishi T, Kanemura Y, Maruno $M$ and Yoshimine T: Quantitative analysis of glioma cell invasion by confocal laser scanning microscopy in a novel brain slice model. Biochem Biophys Res Commun 269: 513-520, 2000.

4. Zhou X, Zhan W, Bian W, Hua L, Shi Q, Xie S, Yang D, Li Y, Zhang X, Liu G and Yu R: GOLPH3 regulates the migration and invasion of glioma cells though RhoA. Biochem Biophys Res Commun 433: 338-344, 2013.

5. Hao K, Tian XD, Qin CF, Xie XH and Yang YM: Hedgehog signaling pathway regulates human pancreatic cancer cell proliferation and metastasis. Oncol Rep 29: 1124-1132, 2013.
6. Qin CF, Hao K, Tian XD, Xie XH and Yang YM: Combined effects of EGFR and Hedgehog signaling pathway inhibition on the proliferation and apoptosis of pancreatic cancer cells. Oncol Rep 28: 519-526, 2012.

7. Fuller K, O'Connell JT, Gordon J, Mauti O and Eggenschwiler J: Rab23 regulates Nodal signaling in vertebrate left-right patterning independently of the Hedgehog pathway. Dev Biol 391: 182-195, 2014.

8. Ju B, Chen W, Spitsbergen JM, Lu J, Vogel P, Peters JL, Wang YD, Orr BA, Wu J and Henson HE: Activation of Sonic hedgehog signaling in neural progenitor cells promotes glioma development in the zebrafish optic pathway. Oncogenesis 3: e96, 2014.

9. Onishi $\mathrm{H}$ and Katano M: Hedgehog signaling pathway as a new therapeutic target in pancreatic cancer. World $\mathrm{J}$ Gastroenterol 20: 2335-2342, 2014.

10. Matsushita S, Onishi H, Nakano K, Nagamatsu I, Imaizumi A, Hattori M, Oda Y, Tanaka M and Katano M: Hedgehog signaling pathway is a potential therapeutic target for gallbladder cancer. Cancer Sci 105: 272-280, 2014.

11. Chen Q, Gao G and Luo S: Hedgehog signaling pathway and ovarian cancer. Chin J Cancer Res 25: 346-353, 2013.

12. Wan J, Zhou J, Zhao H, Wang M, Wei Z, Gao H, Wang Y and Cui H: Sonic hedgehog pathway contributes to gastric cancer cell growth and proliferation. Biores Open Access 3: 53-59, 2014.

13. Zhu W, You Z, Li T, Yu C, Tao G, Hu M and Chen X: Correlation of hedgehog signal activation with chemoradiotherapy sensitivity and survival in esophageal squamous cell carcinomas. Jpn J Clin Oncol 41: 386-393, 2011.

14. Aparna M, Rao L, Kunhikatta V and Radhakrishnan R: The role of MMP-2 and MMP-9 as prognostic markers in the early stages of tongue squamous cell carcinoma. J Oral Pathol Med 44: 345-352, 2015.

15. Zhou M, Qin S, Chu Y, Wang F, Chen L and Lu Y: Immunolocalization of MMP-2 and MMP-9 in human rheumatoid synovium. Int J Clin Exp Pathol 7: 3048-3056, 2014.

16. Yang W, Li Q and Pan Z: Sphingosine-1-phosphate promotes extravillous trophoblast cell invasion by activating MEK/ERK/MMP-2 signaling pathways via S1P/S1PR1 axis activation. PLoS One 9: e106725, 2014.

17. Liu WH and Chang LS: Caffeine induces matrix metalloproteinase-2 (MMP-2) and MMP-9 down-regulation in human leukemia U937 cells via Ca2+/ROS-mediated suppression of ERK/c-fos pathway and activation of p38 MAPK/c-jun pathway. J Cell Physiol 224: 775-785, 2010.

18. Kang MH, Oh SC, Lee HJ, Kang HN, Kim JL, Kim JS and Yoo YA: Metastatic function of BMP-2 in gastric cancer cells: The role of PI3K/AKT, MAPK, the NF- $\kappa$ B pathway, and MMP-9 expression. Exp Cell Res 317: 1746-1762, 2011.

19. Du WZ, Feng Y, Wang XF, Piao XY, Cui YQ, Chen LC, Lei XH, Sun X, Liu X, Wang HB, et al: Curcumin suppresses malignant glioma cells growth and induces apoptosis by inhibition of SHH/GLI1 signaling pathway in vitro and vivo. CNS Neurosci Ther 19: 926-936, 2013.

20. Wang Y, Chen L, Bao Z, Li S, You G, Yan W, Shi Z, Liu Y, Yang P, Zhang W, et al: Inhibition of STAT3 reverses alkylator resistance through modulation of the AKT and $\beta$-catenin signaling pathways. Oncol Rep 26: 1173-1180, 2011.

21. Morgenroth A, Vogg AT, Ermert K, Zlatopolskiy B and Mottaghy FM: Hedgehog signaling sensitizes glioma stem cells to endogenous nano-irradiation. Oncotarget 5: 5483-5493, 2014.

22. Dixit D, Ghildiyal R, Anto NP, Ghosh S, Sharma V and Sen E: Guggulsterone sensitizes glioblastoma cells to Sonic hedgehog inhibitor SANT-1 induced apoptosis in a Ras/NFאB dependent manner. Cancer Lett 336: 347-358, 2013.

23. Das G, Shiras A, Shanmuganandam K and Shastry P: Rictor regulates MMP-9 activity and invasion through Raf-1-MEK-ERK signaling pathway in glioma cells. Mol Carcinog 50: 412-423, 2011.

24. Senft C, Priester M, Polacin M, Schröder K, Seifert V, Kögel D and Weissenberger J: Inhibition of the JAK-2/STAT3 signaling pathway impedes the migratory and invasive potential of human glioblastoma cells. J Neurooncol 101: 393-403, 2011.

25. Zhao Y, Xiao A, Dipierro CG, Abdel-Fattah R, Amos S, Redpath GT, Carpenter JE, Pieper RO and Hussaini IM: H-Ras increases urokinase expression and cell invasion in genetically modified human astrocytes through Ras/Raf/MEK signaling pathway. Glia 56: 917-924, 2008. 
26. Porta $\mathrm{C}$ and Figlin RA: Phosphatidylinositol-3-kinase/Akt signaling pathway and kidney cancer and the therapeutic potential of phosphatidylinositol-3-kinase/Akt inhibitors. J Urol 182: 2569-2577, 2009.

27. Zhang E, Feng X, Liu F, , Zhang P2, Liang J and Tang X: Roles of PI3K/Akt and c-Jun signaling pathways in human papillomavirus type 16 oncoprotein-induced HIF-1 $\alpha$, VEGF and IL-8 expression and in vitro angiogenesis in non-small cell lung cancer cells. PLoS One 9: e103440, 2014.

28. Mazzoletti M and Broggini M: PI3K/AKT/mTOR inhibitors in ovarian cancer. Curr Med Chem 17: 4433-4447, 2010.
29. Yoo YA, Kang MH, Lee HJ, Kim BH, Park JK, Kim HK, Kim JS and Oh SC: Sonic hedgehog pathway promotes metastasis and lymphangiogenesis via activation of Akt, EMT and MMP-9 pathway in gastric cancer. Cancer Res 71: 7061-7070, 2011.

30. Morton JP, Mongeau ME, Klimstra DS, Morris JP, Lee YC, Kawaguchi Y, Wright CV, Hebrok M and Lewis BC: Sonic hedgehog acts at multiple stages during pancreatic tumorigenesis. Proc Natl Acad Sci USA 104: 5103-5108, 2007. 